\title{
A UNIFORM GENERALIZED SCHOENFLIES THEOREM
}

\section{BY PERRIN WRIGHT ${ }^{1}$}

\author{
Communicated by O. G. Harrold, February 13, 1968
}

The generalized Schoenflies theorem of M. Brown [2], [3] can be restated in the following way: If $S^{n-1}$ is the equator of $S^{n}$, then any locally flat embedding $f: S^{n-1} \rightarrow S^{n}$ can be extended to a homeomorphism $F: S^{n} \rightarrow S^{n}$.

The purpose of this paper is to show that, if $n \geqslant 5$, the extension $F$ can be constructed in a controlled manner; in particular, if $f: S^{n-1} \rightarrow S^{n}$ is close to the inclusion embedding, then $F: S^{n} \rightarrow S^{n}$ can be chosen to be close to the identity homeomorphism. Consequently if, $f, g: S^{n-1} \rightarrow S^{n}$ are locally flat embeddings, $n \geqq 5$, and $f$ is close to $g$, then there is a homeomorphism $H: S^{n} \rightarrow S^{n}$ which is close to the identity such that $H f=g$.

Let $S^{n-1}$ denote the unit sphere in $E^{n}, B^{n}$ the unit ball, and $O$ the origin. If $x, y$ belong to $E^{n}-O$, let $\theta(x, y)$ denote the angle in radians between the line segments $O x$ and $O y$, measured such that $0 \leqq \theta(x, y) \leqq \pi$. The distance between $x$ and $y$ under the Euclidean metric will be denoted by dist $(x, y)$. If $A$ is a subset of $E^{n}-O$, the angular diameter of $A$, written $\theta \operatorname{diam} A$, is defined to be $\sup _{x, y \in A} \theta(x, y)$. This is significant whenever $A$ lies in a half-space.

Now suppose $S$ is a locally flatly embedded $(n-1)$-sphere in $E^{n}$ which approximates the standard sphere $S^{n-1}$. Suppose $\phi: S^{n-1}$ $\times[0,1] \rightarrow \mathrm{Cl}($ Ext $S)$ is a collar on $S$ in $\mathrm{Cl}($ Ext $S)$. If the collar is small, then the $\theta$-diameter of each fiber $\phi(x \times[0,1])$ is also small. The object of Lemma 2 is to push the collar outward, leaving $S$ fixed, so that its two boundary components are separated by a round sphere with center at $O$, and so that the $\theta$-diameter of each fiber remains small. The precise statement is as follows.

LEMma 2. If $f: S^{n-1} \rightarrow E^{n}, n \geqq 5$, is a locally flat embedding such that for all $x \in S^{n-1}, \theta(x, f(x))<\epsilon$, where $\epsilon<\pi / 7$, then there is an embedding $F: S^{n-1} \times[0,1] \rightarrow \mathrm{Cl}\left(\operatorname{Ext} f\left(S^{n-1}\right)\right)$ such that:

(1) $F(x, 0)=f(x)$,

(2) $F\left(S^{n-1} \times 0\right)$ and $F\left(S^{n-1} \times 1\right)$ are separated by some round sphere with center at $O$,

(3) For all $x \in S^{n-1}, t \in[0,1], \theta(x, F(x, t))<13 n \epsilon / 2+15 \epsilon$.

\footnotetext{
1 Work on this paper was supported by the National Science Foundation under NSF G5458.
} 
The proof of Lemma 2 requires four auxiliary lemmas. We begin with a collar $\phi$ on $S=f\left(S^{n-1}\right)$, and let $U=\phi\left(S^{n-1} \times(0,1)\right)$. Then $U$ is an open subset of Ext $S$. We further assume $\theta(x, \phi(x, t))<\epsilon$ for all $x$ and $t$. Lemma A states that any complex in Ext $S$ can be pulled into $U$ (in the sense of [1]) by a homotopy in Ext $S$ whose orbits have $\theta$-diameter at most $9 \epsilon$. Lemma B states that any complex in Ext $S$ can be disentangled from $S$, i.e., pulled into the exterior of some round sphere $\boldsymbol{\Sigma}$ outside $S$, by a homotopy in Ext $S$ whose orbits have $\theta$ diameter less than $4 \epsilon$. The condition that $\theta(x, f(x))<\epsilon$ for all $x \in S^{n-1}$ insures that the "folds" in $S$ are small, and hence any point of Ext $S$ may be moved into $U$ or outside $\Sigma$ along a path of small $\theta$-diameter. The condition $\epsilon<\pi / 7$ is a purely artificial one which makes the proofs work.

Lemmas $1 \mathrm{~A}$ and $1 \mathrm{~B}$ are radial engulfing lemmas. The engulfings proceed along the orbits of the homotopies guaranteed by Lemmas $\mathrm{A}$ and $\mathrm{B}$. The proofs of these lemmas are almost identical to the proof of Engulfing Theorem A of [1], and their functions are comparable to those of Lemmas 1 and 2 of [5].

Finally, the proof of Lemma 2 is accomplished in the manner of Lemma 9.1 of [6].

If $S_{1}$ and $S_{2}$ are disjoint locally flat $(n-1)$-spheres in $E^{n}, S_{1} \subset \operatorname{Int} S_{2}$, and if there is a stable homeomorphism $h: E^{n} \rightarrow E^{n}$ such that $h\left(S_{1}\right)$ $=S_{2}$, then $S_{1}$ and $S_{2}$ cobound an annulus (Theorem 10.3 of [4]). We next strengthen a special case of this theorem.

Let $\bar{S}$ be a sphere concentric with $S^{n-1}$. Let $x$ denote the point of $\bar{S}$ which is coradial with $x \in S^{n-1}$. Introduce the following notation: if $y \in E^{n}-O$ and $L$ is a real number such that $\|y\|+L>0$, then $y+L$ denotes the unique point of $E^{n}$ which is coradial with $y$ and has norm $\|y\|+L$,

If $f: S^{n-1} \rightarrow E^{n}$ and $\bar{f}: \bar{S} \rightarrow E^{n}$ are embeddings, we say that $f$ and $\bar{f}$ are parallel if there is a real number $L$ such that for all $x \in S^{n-1}, f(x)$ $=\bar{f}(\vec{x})+L$.

Clearly any two disjoint parallel spheres are stably equivalent, hence cobound an annulus. Lemma 3 states that this annulus can be coordinatized so that the $\theta$-diameters of the fibers are directly proportional to the $\theta$-deviation of $f$ itself.

LEMMa 3. Let $\bar{S}$ be a sphere concentric with $S^{n-1}$, of radius less than 1 . Let $A$ be the annulus between $\bar{S}$ and $S^{n-1}$. Let $0<\epsilon<\pi / 7$, and let $f: S^{n-1} \rightarrow E^{n}, n \geqq 5$, be a locally flat embedding such that $\theta(x, f(x))<\epsilon$ for all $x \in S^{n-1}$. Suppose $\bar{f}: \bar{S} \rightarrow \operatorname{Int} f\left(S^{n-1}\right)$ is an embedding which is parallel to $f$. Then there is an embedding $F: A \rightarrow E^{n}$ such that: 
(1) $F \mid S^{n-1}=f$,

(2) $F \mid \bar{S}=\bar{f}$,

(3) $\theta(y, F(y))<(39 / 2) n \epsilon+45 \epsilon$, for all $y \in A$.

To prove Lemma 3, apply Lemma 2 to obtain an annulus in $\mathrm{Cl}\left(\operatorname{Ext} f\left(S^{n-1}\right)\right)$ which satisfies the conclusion of Lemma 2. Call this annulus $R_{1}$, and denote the annulus between $f\left(S^{n-1}\right)$ and $\bar{f}(\bar{S})$ by $R_{2}$. Using the fact that Int $R_{1}$ contains a round sphere with center at $O$, push $R_{1}$ onto $R_{1} \cup R_{2}$ by a radial homeomorphism of $E^{n}$. This does not alter the $\theta$-diameters of the fibers of $R_{\mathbf{1}}$. Next, map $R_{1} \cup R_{\mathbf{2}}$ homeomorphically onto $R_{2}$ by utilizing the annular structure on $R_{1}$. This at worst triples the $\theta$-diameters of fibers. The result of these maps gives an annular structure on $R_{2}$ satisfying Lemma 3.

\section{The main theorems.}

TheOREM 1. If $n \geqq 5$, and $f: S^{n-1} \rightarrow E^{n}$ is a locally flat embedding such that $\theta(f(x), x)<\epsilon$ and $\operatorname{dist}(f(x), x)<\epsilon$ for all $x \in S^{n-1}$, then $f$ can be extended to an embedding $F: B^{n} \rightarrow E^{n}$ such that dist $(F(x), x)$ $<39 n \epsilon / 2+48 \epsilon$.

Corollary 1. For each $\eta>0$, there is a $\delta>0$ such that each locally flat $\delta$-embedding of $S^{n-1}$ into $E^{n}, n \geqq 5$, can be extended to an $\eta$-embedding of $B^{n}$ into $E^{n}$.

The proof of Theorem 1 is outlined as follows. Partition $B^{n}$ into annuli $A_{i}$ of thickness $2 \epsilon$ together with a small ball $B_{*}$ in the center. Partition $\mathrm{Cl}\left(\operatorname{Int} f\left(S^{n-1}\right)\right)$ into annular regions $R_{i}$, together with a small cell $C$ about the origin, in such a way that each boundary sphere of each $R_{i}$ is parallel to $f\left(S^{n-1}\right)$ and the parallel distance between any two consecutive spheres (i.e., the constant $L$ of the definition of parallel embeddings) is $2 \epsilon$. Obtain a 1-1 correspondence between the $A_{i}$ and the $R_{i}$ by omitting the innermost $A_{i}$ or $R_{i}$, if necessary. Use Lemma 3 to map the outermost annulus $A_{0}$ homeomorphically on to the outermost region $R_{0}$. (We assume $\epsilon<\pi / 7$, for if not, Theorem 1 is certainly true.) Then it is possible to map each $A_{i}$ onto the corresponding $R_{i}$ by copying the map $f \mid A_{0}$. This procedure is well defined on $A_{i} \cap A_{i+1}$, because of the parallel condition. Finally, map $B_{*}$ homeomorphically onto $C$ in any fashion, extending the map $F \mid \dot{B}_{*}$.

For points $y \in A_{i},|\|y\|-\|F(y)\||<3 \epsilon$ and $\theta(y, F(y))<(39 / 2) n \epsilon$ $+45 \epsilon$. Since $\|y\| \leqq 1$, dist $(y, F(y))<(39 / 2) n \epsilon+48 \epsilon$. For points $y \in B_{*}$ no control is necessary because $B_{*} \cup C$ has diameter less than $7 \epsilon$.

Now consider $S^{n-1}$ to be the equator of $S^{n}$. Theorems 2 and 3 follow from Corollary 1. 
THEOREM 2. Let $n \geqq 5, \eta>0$. There is a $\delta>0$ such that any locally flat $\delta$-embedding $f: S^{n-1} \rightarrow S^{n}$ can be extended to a $\eta$-homeomorphism $F: S^{n} \rightarrow S^{n}$.

ThEOREM 3. Let $n \geqq 5, \eta \geqq 0$. Let $g: S^{n-1} \rightarrow S^{n}$ be any locally flat embedding. There exists $a \delta>0$ such that if $f: S^{n-1} \rightarrow S^{n}$ is any locally flat embedding satisfying $\operatorname{dist}(f(x), g(x))<\delta$, then there is an $\eta$-homeomorphism $H: S^{n} \rightarrow S^{n}$ such that $H f=g$.

These results, together with those of Connell [5] and Bing [1], can be used to show that the problem of approximating homeomorphisms of $S^{n}, n \geqq 5$, by p.w.l. ones is equivalent to approximating locally flat embeddings of $(n-1)$-spheres by p.w.l. ones.

\section{REFERENCES}

1. R. H. Bing, Radial engulfing, Notes of address given at Michigan State University Conference on Topology, 1967.

2. M. Brown, Locally flat embeddings of topological manifolds, Ann. of Math. 75 (1962), 331-341.

3. - A proof of the generalized Schoenflies theorem, Bull. Amer. Math. Soc. 66 (1960), 74-76.

4. M. Brown and H. Gluck, Stable structures on manifolds. I: Homeomorphisms of $S^{n}$, Ann. of Math. 79(1964), 1-17.

5. E. H. Connell, Approximating stable homeomorphisms by piecewise linear ones, Ann. of Math. 78(1963), 326-338.

6. John Stallings, On topologically unknotted spheres, Ann. of Math. 77(1963), 490-503.

Florida State University 